\title{
Miniature Solid Tunable Lenses and Their Applications: A Review
}

\author{
Yongchao Zou, Fook Siong Chau, and Guangya Zhou* \\ Micro and Nano Systems Initiative, Department of Mechanical Engineering, \\ National University of Singapore, 9 Engineering Drive 1, Singapore 117575
}

(Received August 1, 2016; accepted October 26, 2016)

Keywords: optical design, lenses, optical micro-electromechanical devices, freeform optics, imaging systems

We present a systematic review of solid tunable lenses based on the Alvarez-Lohmann principle, which mainly focuses on the optimization, miniaturization, and integration of such solid tunable lenses in miniature applications. The progress of the modelling and design methods of such solid tunable lenses for optimum performance is summarized first. The development of various solid tunable lenses achieved using diverse driving mechanisms and different optimization methods is then illustrated by examples from current literature. The mechanisms, performance, and practicability of all these prototypes are presented in detail. The applications of such solid tunable lenses in miniature imaging systems are finally reviewed. Discussions of the problems and bottlenecks encountered in current research work and perspectives for future work in this field are given.

\section{Introduction}

Optical systems are endowed with the capability of autofocus or zooming by the well-known method of mechanically or electrically moving one or more lenses along the optical axis, changing the effective focal length of the lens system. Such a configuration obviously faces the problems of bulky structures and complicated support and drive mechanisms, and therefore is not suitable for applications in modern miniature systems. To scale down conventional adjustable-focus or zoom lens systems for miniature applications, over the past few decades, much effort has been spent on developing various compact tunable lenses, including diverse liquid lenses and solid tunable lenses.

To adjust the focal length of a liquid lens, one needs to change either the geometric shape of the lens or the refractive index distribution of the lens material.(1,2) There have been extensive trials to achieve liquid lenses with deformable geometric shapes employing various driving mechanisms. ${ }^{(3-16)}$ They include diverse flexible-membrane lenses, electrowetting-based lenses, and dielectrophoretic lenses. ${ }^{(17-29)}$ Rather than modulating the geometric shape of the lens, one can also choose to vary the refractive index of the lens material. One of the typical examples is liquid-crystal (LC) lenses. ${ }^{(30-36)}$ Some other methods of modifying the refractive index of the lens include subjecting the liquid to an external electrical field, acoustic field, mechanical strain, or temperature field, as the optical properties of most of the materials are related to these external fields. ${ }^{(37)}$ We cannot deny the fact

"Corresponding author: e-mail: mpezgy@nus.edu.sg http://dx.doi.org/10.18494/SAM.2017.1440 
that most of the attention has been paid to liquid lenses over the past years in this field. However, it is widely noted that liquid lenses face a series of challenges, including complex fabrication process, performance instability, possible leakage, and evaporation. All these issues greatly limit their applications in practical miniature imaging systems.

Solid tunable lenses, which involve pure solid-state materials in the lens configuration, have drawn increasing attention in recent years as they offer an effective way to solve the aforementioned issues encountered by the liquid lenses. Thanks to the absence of liquid materials, solid tunable lenses perform stably and can be fabricated by a relatively simple process without concerns of leakage or evaporation issues, and thereby show greater potential in practical applications. The first kind of solid tunable lenses are realized by simply deforming the solid lens by a thermal effect or external strain. ${ }^{(38-41)}$ However, because they are constrained by the maximum deformation that can be achieved by either thermal actuation or external strains, such a type of solid tunable lenses can only provide a limited tuning range of focal lengths, which is obviously far from the requirement of most practical applications.

The second kind of solid tunable lenses are designed on the basis of the Alvarez-Lohmann principle, and focal length tuning is accomplished by slightly shifting two cubic lens elements with respect to each other along the direction perpendicular to the optical axis. ${ }^{(42,43)}$ Such solid tunable lenses can provide large focal-length tuning ranges with compact structures. Furthermore, the fabrication process of the lens elements is relatively simple and the imaging performance of such solid tunable lenses is satisfactory and stable. Hence, they show a more promising future in various miniature applications compared with their counterparts. In this paper, the progress of such solid tunable lenses is reviewed in detail, including the design methods of the lens elements to gain optimum performance, various prototypes achieved with different driving mechanisms, and their applications in miniature imaging systems. Discussions of the problems and bottlenecks encountered in current research and perspectives for future work in this field are also given.

\section{Design Methods for Optimum Lens Performance}

\subsection{Alvarez-Lohmann principle}

As shown in Fig. 1, the solid tunable lens based on the Alvarez-Lohmann principle consists of two elements, each of which has one flat surface and one cubic surface. The sag (thickness along the $z$ direction) of the cubic surface is given by ${ }^{(42-44)}$

$$
z=A_{x} x^{3}+A_{x}^{\prime} y x^{2}+A_{y} y^{3}+A_{y}^{\prime} y x^{2}+B x^{2}+C x y+D y^{2}+E x+F y+G
$$

where $z$ is the thickness of the lens element at the point determined by the coordinate $(x, y) . A_{x}, A_{x}^{\prime}$ $A_{y}, A_{y}^{\prime}, B, C, D, E, F$, and $G$ are the coefficients of the terms in the cubic polynomial. As the secondorder terms give the optical power or astigmatism in the phase delay, the coefficients $B, C$, and $D$ are normally set to zero to simplify the analysis. In the Alvarez configuration, $A_{x}^{\prime}$ and $A_{y}$ are set to zero while in the Lohmann configuration, $A_{x}^{\prime}$ and $A_{y}^{\prime}$ are set to zero. Hence, the surface-governing equation, Eq. (1), is changed to the following one for the two configurations.

$$
\begin{aligned}
& \text { Alvarez configuration: } z=A_{x} x^{3}+A_{y}^{\prime} y x^{2}+E x+F y+G \\
& \text { Lohmann configuration: } z=A_{x} x^{3}+A_{y} y^{3}+E x+F y+G
\end{aligned}
$$




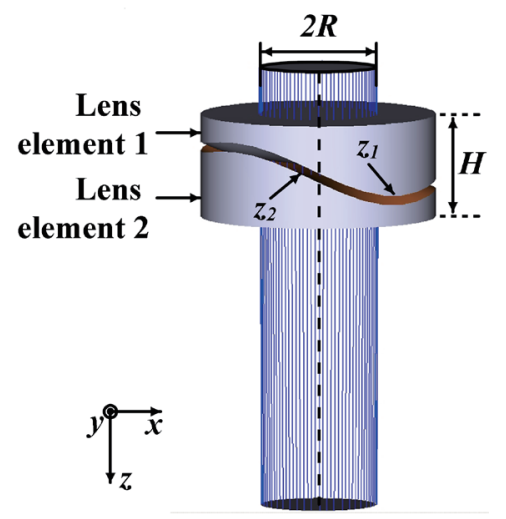

(a)

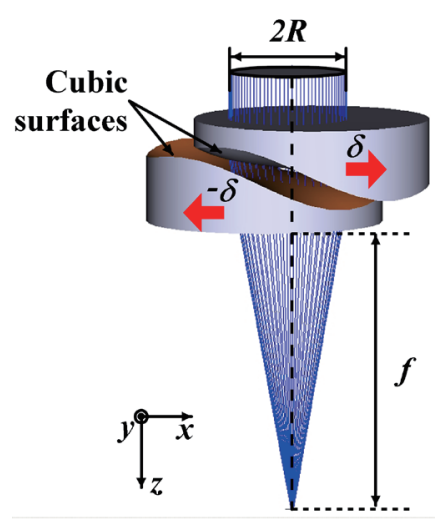

(b)

Fig. 1. (Color online) Schematics of a solid tunable lens based on the Alvarez-Lohmann principle when the two lens elements are (a) perfectly aligned and (b) shifted with lateral displacements.

The optical power variation is introduced in the Alvarez configuration when the two lens elements are shifted with a lateral displacement $\delta$ in the $x$ and $-x$ directions. The overall thickness of the lens configuration is then determined by

$$
\begin{aligned}
t_{\text {overall }}= & z_{1}(x-\delta)+H-z_{2}(x+\delta) \\
= & A_{x}(x-\delta)^{3}+A_{y}^{\prime}(x-\delta) y^{2}+E(x-\delta)+F y+G \\
& +H-\left[A_{x}(x+\delta)^{3}+A_{y}^{\prime}(x+\delta) y^{2}+E(x+\delta)+F y+G\right],
\end{aligned}
$$

where $H$ is a constant representing the overall thickness from the top flat surface of element 1 to the bottom flat surface of element 2. By a simple mathematical derivation, we can obtain

$$
t_{\text {overall }}=-6 A_{x} \delta x^{2}-2 A_{y}^{\prime} \delta y^{2}+H-2 E \delta-2 A_{x} \delta^{3} .
$$

To generate a pure optical power, the coefficient $A_{x}$ is set to be equal to $A_{y}^{\prime} / 3$ in the Alvarez configuration. Therefore, the overall thickness is given by

$$
t_{\text {overall }}=-2 A_{y}^{\prime} \delta\left(x^{2}+y^{2}\right)+H-2 E \delta-\frac{2}{3} A_{y}^{\prime} \delta^{3},
$$

where $H-2 E \delta-2 A_{y}^{\prime} \delta^{3} / 3$ is a constant, contributing nothing but a piston to the lens thickness. If the refractive index of the material is $n$, note that the overall optical phase delay (OPD) generated by such a lens configuration is determined to be

$$
O P D=-2 A_{y}^{\prime} \delta(n-1)\left(x^{2}+y^{2}\right)+C,
$$

where $C$ is a piston constant. Such an OPD offers a spherical optical power, which makes the twoelement configuration equivalent to an optical lens with the focal length determined by

$$
f=1 /\left[4 A_{y}^{\prime} \delta(n-1)\right]
$$


From Eq. (7), we can note that the focal length is related to the lateral displacement $\delta$; this means that the focal length is tunable with the dynamic variation of the lateral position of the two lens elements. Furthermore, note that the value of $A_{y}$ ' controls the "speed" of tuning.

By a similar mathematical derivation process as that from Eq. (3) to Eq. (7), we can find that, in the Lohmann configuration, a lateral displacement of the two elements in the $x$ or $y$ direction produces a cylindrical optical power. In order to generate a spherical optical power, the two elements must be moved with the same displacement in both $x$ and $y$ directions, and the coefficients must meet the conditions of $A_{x}=A_{y}$ and $E=F$. Note that applying a 45-degree coordinate rotation to the Lohmann sag equation gives the Alvarez sag equation multiplied by $\sqrt{2} / 2$. Hence, the characteristics of these two lens configurations are inherently the same. ${ }^{(45)}$ The only difference is the scaling factor $\sqrt{2} / 2$, which means that the Alvarez lens requires a smaller lateral displacement than the Lohmann one to produce the same optical power if the coefficient of the third-order term in Eq. (2) is the same. Therefore, in most miniature applications, the Alvarez configuration is adopted since the maximum lateral displacement of the lens elements should be minimized to save space. All the solid tunable lenses discussed in the following sections are designed in accordance with the Alvarez configuration without further explanations.

\subsection{Optimum design of solid tunable lens}

To optimize the performance of the solid tunable lens, we must consider a few issues. Firstly, to implement the solid tunable lens, the two lens elements can be arranged in two different configurations, where either the two cubic surfaces or the two flat surfaces are placed close to each other (namely, the inner cubic configuration or outer cubic configuration, respectively). To guide the lens design, Barbero numerically compared the performances of these two configurations, and concluded that the lens with inner cubic surfaces performs better than the other one. ${ }^{(45)}$

Secondly, one must optimize the coefficient values in the governing equation. As discussed in Ref. 45, the value of $A_{y}^{\prime}$ determines the ratio between the focal length and the lateral displacement and hence controls the speed of focal length tuning. Therefore, the value of $A_{y}^{\prime}$ is determined by the required optical focal-length tuning range and the maximum achieved lateral displacement from the actuators. The constant $G$ is nothing more than a piston determining the center-to-center thickness of the lens element. Theoretically, the effect of $G$ on lens performance is negligible, except the possible scattering in the material. The values of $E$ and $F$ determine the weights of the tilt terms in the governing equation. Alvarez pointed out that the coefficient $E$ in Eq. (2) can be used to reduce the overall lens thickness, which is beneficial to lens performance. ${ }^{(42)}$ Hence, it is concluded that the minimum lens thickness leads to the optimal lens performance. On the basis of this concept, Barbero derived an analytical expression of the coefficient $E$ and regarded it as the optimal choice for the lens design: ${ }^{(45)}$

$$
E=-A_{y}^{\prime} R^{2} / 3,
$$

where $R$ is the radius of the lens element.

However, both Alvarez and Barbero ignored the air gap between the two lens elements in the derivation and assumed the lens element to be an ideal phase plate, but this actually deviates from the practical situation. Zou et al. noted that it is the air gap rather than the lens thickness that should be the dominant factor affecting the lens performance, on the basis of their experimental 
and simulation results. ${ }^{(46)}$ To achieve the optimal lens performance, the air volume between the two lens elements should be minimized in the inner configuration, and hence the optimum value of $E$ is given by

$$
E_{\text {optimal }}= \begin{cases}-\frac{A_{y}^{\prime} \delta_{\max }^{2}}{3}-\frac{g_{0}}{2 \delta_{\max }} & \text { if } \delta_{\max } \leq\left(\frac{3 g_{0}}{4 A_{y}^{\prime}}\right)^{\frac{1}{3}} \\ \left(-\frac{9 A_{y}^{\prime} g_{0}^{2}}{16}\right)^{\frac{1}{3}} & \text { if } \delta_{\min }<\left(\frac{3 g_{0}}{4 A_{y}^{\prime}}\right)^{\frac{1}{3}}<\delta_{\max }, \\ -\frac{A_{y}^{\prime} \delta_{\min }^{2}}{3}-\frac{g_{0}}{2 \delta_{\min }} & \text { if } \delta_{\min } \geq\left(\frac{3 g_{0}}{4 A_{y}^{\prime}}\right)^{\frac{1}{3}}\end{cases}
$$

where $g_{0}$ is the center-to-center distance between the two lens elements at the initial position without any lateral displacement. $\delta_{\min }$ and $\delta_{\max }$ represent the minimum and maximum lateral displacements of the lens element during operation, respectively.

To compare these two methods for coefficient selection, two solid tunable lenses are designed with all the parameters kept the same, except for the value of $E .^{(46)}$ In Fig. 2, the imaging simulation results of these two tunable lenses are shown. Figures 2(a)-2(c) represent the performance of

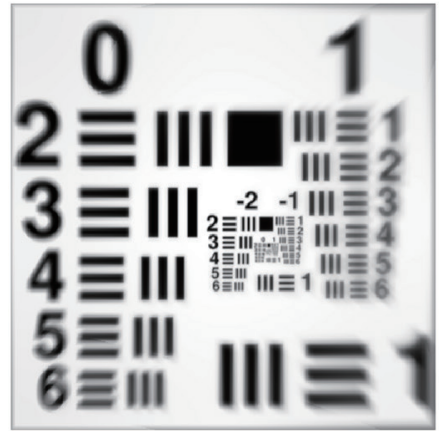

(a)

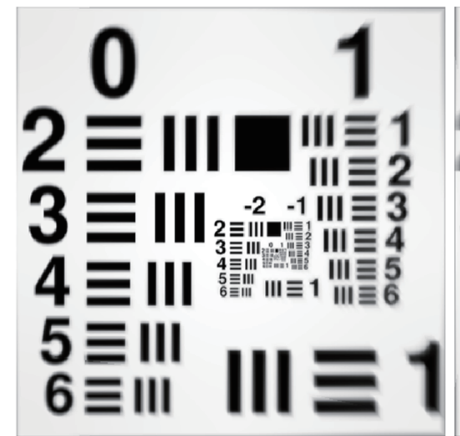

(d)

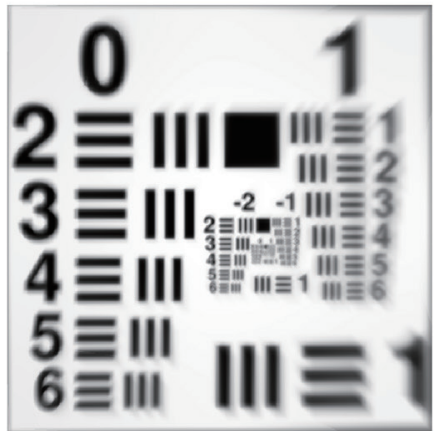

(b)

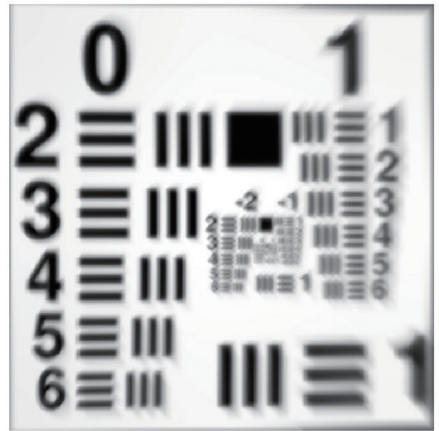

(c)

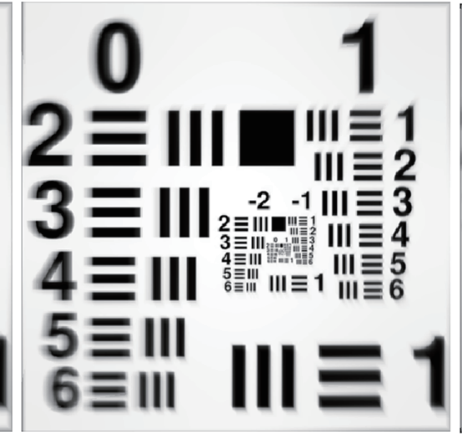

(e)

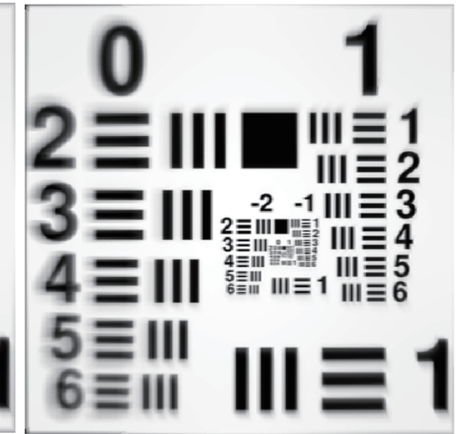

(f)

Fig. 2. Imaging simulation results of the solid tunable lens designed according to (a)-(c) Eq. (8) and (d)-(g) Eq. (9). The lateral displacement of the lens element is set at $0.15 \mathrm{~mm}$ in (a) and (d), $0.30 \mathrm{~mm}$ in (b) and (f), and $0.45 \mathrm{~mm}$ in (c) and $(\mathrm{g})$. All the parameters are kept the same in these two solid tunable lenses, except for the value of $E$. 
the solid tunable lens designed according to Eq. (8), while Figs. 2(d)-2(g) show that of the lens designed according to Eq. (9). Note that the image quality is improved considerably by the latter method.

However, note that both Eqs. (8) and (9) give only a rough solution for the lens optimization problem. To achieve better lens performance, the ray-tracing technique is clearly necessary to optimize the lens surfaces. The coefficient values given by Eq. (8) or (9) could be a good starting point for the optimization process. It is apparent that the degrees of freedom are quite limited in the optimization process if Eq. (2) is used to describe the lens surface. To improve the lens performance, Barbero and Rubinstein modified the surface-governing equation given by Eq. (2) to a more general cubic polynomial plus a standard conic term, as shown below:(47)

$$
z=\frac{c r^{2}}{1+\sqrt{1-(K+1) c r^{2}}}+p_{1} x^{3}+p_{2} y x^{2}+p_{3} y^{2} x+p_{4} y^{3}+p_{5} y x+p_{6} y+p_{7} x
$$

where $r=\sqrt{x^{2}+y^{2}}$, and $c$ and $K$ are the curvature and conic constant of the surface, respectively. $p_{1}$ to $p_{7}$ are the coefficients of the terms in the cubic polynomial. By using this improved model, one has up to nine parameters $\left(c, K\right.$, and $\left.p_{i}\right)$ for each surface in the optimization process, and hence, more degrees of freedom for searching the optimal solution.

In 2014, Grewe et al. further analyzed the aberrations of the Alvarez-Lohmann lenses, and pointed out that the use of polynomials with higher-order terms is feasible to correct aberrations in the solid tunable lenses. ${ }^{(48)}$ Subsequently, Zou et al. and Petsch et al. experimentally demonstrated two miniature solid tunable lenses, respectively, with the freeform surfaces governed by separated extended polynomials with higher orders, as given by ${ }^{(49,50)}$

$$
z=\sum_{m, n=0}^{m, n=Q} a_{n, m} \cdot x^{n} y^{m},
$$

where $a_{n, m}$ is the coefficient of the $(m+n)$ th-order term in the polynomial. $Q$ is the highest order of the polynomial. Zou et al. employed two 6-order polynomials $(Q=6)$ to govern the two freeform surfaces independently, and achieved a diffraction-limited solid tunable lens. ${ }^{(49)}$ Petsch et al. further pointed out that the fourth- and sixth-order aberrations dominate the phase of the transmitted wavefront from the lens. Hence, they used the fifth- and seventh-order thickness terms in the polynomial $(Q=7)$ to compensate these aberrations and even-order terms to minimize the aberrations at the initial position. ${ }^{(50)}$

Rather than the extended polynomials used in Eq. (11) for aberration compensation, other surface description functions proposed recently, including radial basis functions, ${ }^{(51)} \varphi$-polynomials, ${ }^{(52)}$ and their hybrids, ${ }^{(53)}$ could also be used in the governing equation to improve the lens performance. A detailed comparison between these surface-description methods would be helpful to researchers in choosing the right modelling method in future lens designs.

\section{Prototypes of Solid Tunable Lenses with Various Driving Mechanisms}

In recent years, thanks to the rapid development of precision machining technology, the realization of various freeform surfaces has become possible. Over the past few years, both refractive and diffractive solid tunable lenses based on the Alvarez-Lohmann principle have been 
experimentally demonstrated. ${ }^{(54-57)}$ The first solid tunable lens based on the Alvarez principle, working in a diffractive manner, was experimentally achieved using photolithography in 2000.(54) Such a lens consists of two phase plates with 16 phase levels, fabricated by standard multistep photolithography and then tested with an interferometer. The results show that aberrations owing to fabrication and assembly errors are controlled to be under $0.1 \lambda$, which guarantees an excellent performance of the lens applied in laser systems. In 2004, Rege et al. reported a miniaturized scanning microscope based on such solid tunable lenses. ${ }^{(58)}$ The hybrid lenses in a conventional microscope are hypothesized to be replaced by solid tunable lenses in order to miniaturize the microscope. However, no experimental results were presented in that paper due to fabrication challenges.

To the best knowledge of the authors, in 2006, the first materialized transmissive Alvarez lens was reported by Simonov et al. ${ }^{(59)}$ The optical lens elements are made of hydroxyethylmethacrylate (HEMA)/methyl methacrylate (MMA) copolymer processed by diamond lathing. The ultimate purpose of such a lens is to realize an accommodative intraocular lens for use in ophthalmic diagnosis. Similarly, three years later, Schwiegerling and Patela-Toxqui demonstrated the concept of a novel telescope using a pair of Alvarez lenses to achieve a zooming function with fixed object and image positions. ${ }^{(57)}$ In 2008, a micro-Alvarez lens array was reported for the first time by Huang et al. ${ }^{(55)}$ A $5 \times 5$ micro-Alvarez lens array mold was fabricated using a 5-axis ultraprecision diamond machine, and then the lens array was manufactured by an injecting molding process. All measured profiles showed excellent agreement with the design data. Huang et al. introduced an effective method of lens element fabrication and replication. In 2012, Smilie et al. presented an Alvarez lens designed for infrared applications. ${ }^{(56)}$ The lens element is made from germanium and fabricated with the diamond micromilling technology. A custom-built imaging test station is utilized to characterize the performance of the lens. Results show that focal length tuning in the range from 40 to $170 \mathrm{~mm}$ is experimentally achieved.

To integrate such solid tunable lenses into miniature applications, much effort has also been paid to their miniaturization and optimization. As a focus of this paper, the research work in this specific field is discussed in detail in the following section.

\subsection{Miniature solid tunable lenses}

In 2013, Zhou et al. reported the first micro-electromechanical-systems (MEMS)-driven miniature solid tunable lens based on the Alvarez-Lohmann principle, the dimensions of which are controlled to be millimeter order, as shown in Fig. 3(a). ${ }^{(60)}$ The lens elements are fabricated with a diamond turning technology followed by a polydimethylsiloxane (PDMS) replication process while the electrostatic actuators driving the lens elements are prepared by standard silicone-on-insulator (SOI) multi-user-MEMS processes (MUMPs). With a lateral movement of $40 \mu \mathrm{m}$ provided by the actuators, dynamic focal length tuning from 3 to $4.65 \mathrm{~mm}$ is experimentally achieved for the developed miniature solid tunable lens (the aperture size of the lens is around $2 \mathrm{~mm}$ ). However, the imaging quality of the lens is found to be unsatisfactory owing to the rough modelling method and low alignment accuracy.

To improve the lens performance and verify the coefficient selection method proposed as Eq. (9), a miniature solid tunable lens driven by a piezo actuator was developed in 2015, as illustrated in Fig. 3(b). ${ }^{(61)}$ The lens elements are designed on the basis of the aforementioned method and fabricated by a single-point diamond turning technology followed by a PDMS replication process. One compact piezo actuator is employed to provide an initial displacement, which is sequentially 


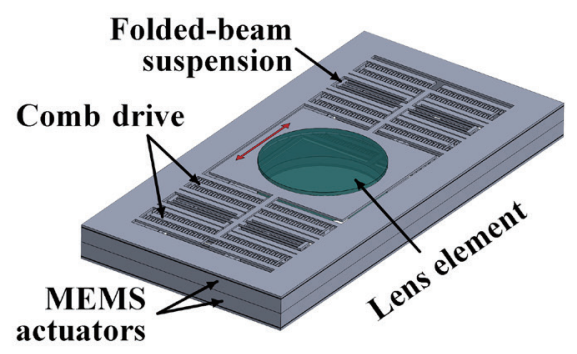

(a)

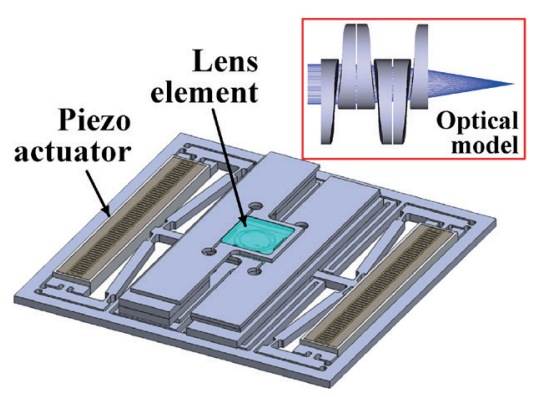

(c)

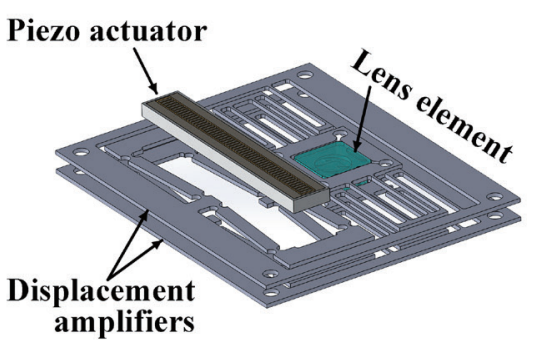

(b)

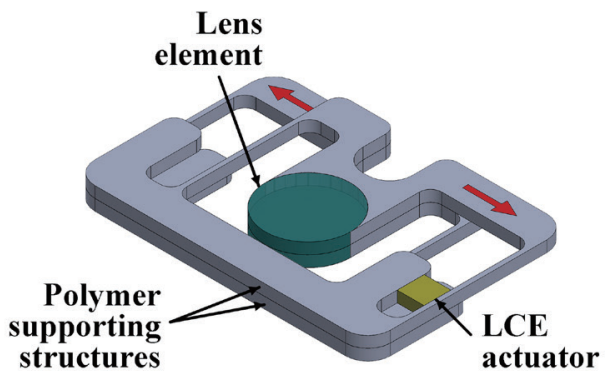

(d)

Fig. 3. (Color online) Schematics of the developed miniature solid tunable lenses. (a) MEMS-driven miniature solid tunable lens, (b) solid tunable lens driven by piezo actuator, (c) multi-element solid tunable lens, and (d) solid tunable lens actuated by artificial muscles. "LCE" is short for "liquid crystal elastomer" in the figure. Note that the schematics here are for illustration purposes only and are not scaled 1:1 from the original devices reported in the literature.

amplified by a mechanical displacement amplifier. The results show that the designed solid tunable lens offers a dynamic focal-length tuning range of about 2.3 times (from 28 to $65 \mathrm{~mm}$ ). The images formed by the lens show superior quality to the ones formed by the conventional miniature Alvarez lenses. The results reveal that the coefficient selection method given by Eq. (9) is effective to improve the lens performance.

Note that the maximum optical power and its tuning range are both limited in conventional solid tunable lenses designed in accordance with the Alvarez-Lohmann principle owing to the limited maximum displacements provided by miniature actuators. To solve this problem, a novel multi-element solid tunable lens was proposed and developed by Zou et al. ${ }^{(62)}$ More specifically, as presented in Fig. 3(c), multiple pairs of lens elements are stacked and driven synchronously by two piezo actuators integrated with mechanical displacement amplification mechanisms. Thanks to the accumulation of the optical powers from the stacked elements, a much higher optical power and greater tuning range, together with satisfactory image performance, are achievable. A fourelement solid tunable lens is experimentally demonstrated in comparison with a conventional twoelement one. The results show that the four-element lens has an optical-power tuning range from 50.9 to 94.1 diopters while for the two-element lens, the optical power is tuned from 25.3 to 46.4 diopters. The results suggest that the proposed multi-element tunable lens might offer a practical way to achieve large optical power variations with small stroke microactuators, thereby facilitating the miniaturization of such lenses.

In 2016, Petsch et al. reported an ultrathin solid tunable lens design based on the AlvarezLohmann principle, as illustrated in Fig. 3(d) schematically. ${ }^{(50)}$ The lens surface is governed by a 
7th-order polynomial and optimized by the ray-tracing technique. The lens element is actuated by liquid crystal artificial muscle actuators. ${ }^{(63)}$ The lens elements and the supporting mechanisms are fabricated by a polymer molding process. The results show that in the designed solid tunable lens, the focal length can be changed by $3.3 \mathrm{~mm}$ from the original focal length of $28.4 \mathrm{~mm}$. However, the imaging quality of such a lens is not satisfactory. Possible reasons may include fabrication errors arising during the modelling process and alignment errors.

Note that various prototypes of such solid tunable lenses have been demonstrated, but further efforts are still necessary to improve the actuator designs and integration methods in order to further scale down the devices. In addition, novel alignment mechanisms of the lens elements are also desirable to improve the lens performance and simplify the assembly process.

\subsection{Variations of solid tunable lens based on Alvarez-Lohmann principle}

Inspired by the Alvarez-Lohmann principle, Zou et al. proposed a novel miniature solid tunable dual-focus lens, which is designed using freeform optical surfaces and driven by one MEMS rotary actuator. ${ }^{(64)}$ As shown in Fig. 4(a), such a lens consists of two optical elements, each of which has a flat surface and one freeform surface governed by the following equation and optimized by raytracing technology.

$$
z=\frac{c r^{2}}{1+\sqrt{1-(1+k) c^{2} r^{2}}}+\sum_{i=1}^{N} B_{i} Z_{i}(r, \theta)+ \begin{cases}A r^{2}(\theta+\phi) & \pi \geq \theta \geq 0 \\ A r^{2}(2 \pi-\theta+\phi) & 2 \pi>\theta>\pi\end{cases}
$$

Here, $r$ and $\theta$ are the radii and azimuth, respectively, in the polar coordinate system. $c$ and $k$ present the curvature and conic constant of the surface. $B_{i}$ is the weight of the $i$ th Zernike polynomial $Z_{i}(r, \theta)$. A is a constant controlling the "speed" of tuning and $\phi$ is an azimuth constant determining the initial focal length of the lens without any rotation.

By changing the relative rotation angle instead of the lateral positions of the two lens elements, the lens configuration can form double foci with corresponding focal lengths simultaneously varied, resulting in a tunable dual-focus effect, as presented in Fig. 4(b). Experimental results show that one of the focal lengths is tuned from about 30 to $20 \mathrm{~mm}$, while the other one is varied from about 30 to $60 \mathrm{~mm}$ with a maximum rotation angle of about $8.2^{\circ}$ provided by the MEMS thermal actuator.

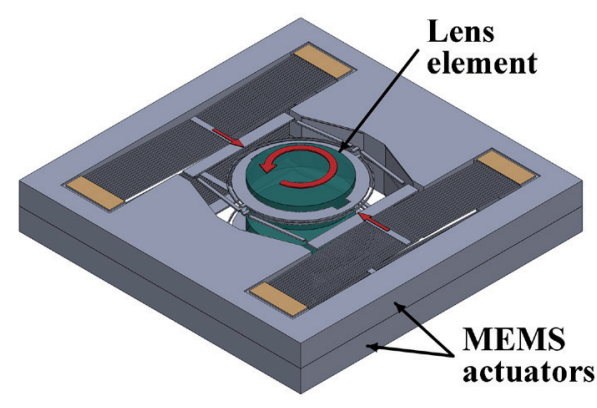

(a)

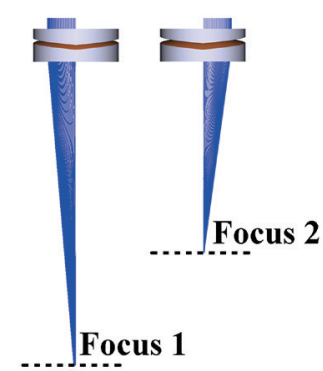

(b)

Fig. 4. (Color online) (a) Schematics of the solid tunable dual-focus lens and (b) ray-tracing result of the dualfocus lens showing its working principle. 
Such a kind of solid tunable dual-focus lens would be useful in various optical systems, including laser cutting systems, microscopy systems, and interferometer-based surface profilers. Further studies in this field would be of great interest as more novel devices become achievable through the variations of the Alvarez-Lohmann principle.

\section{Applications of Solid Tunable Lenses in Miniature Imaging Systems}

To explore the applications of such miniature solid tunable lenses, a miniature adjustablefocus endoscope was developed by Zou et al. in 2015.(49) As shown in Fig. 5, such an endoscope consists of a solid tunable lens for optical power tuning, two slender piezoelectric benders to laterally move the lens elements perpendicular to the optical axis, and an image fiber bundle for image transmission. Dynamic tuning of optical powers from about 135 to about 205 diopters is experimentally achieved by the solid tunable lens, which contains two freeform surfaces governed by 6-degree polynomials and optimized by ray tracing. Results show that there is no obvious distortion or blurring in the images obtained, and the recorded resolution of the lens reaches about 30 line pairs per $\mathrm{mm}$. Three test targets located at various object distances of 20,50, and $150 \mathrm{~mm}$ are focused individually by the endoscope by applying different driving DC voltages to demonstrate its adjustable-focus capability.

Recently, a variable-focus camera module integrated with one MEMS-driven solid tunable lens was demonstrated by Zhang et al. ${ }^{(65)}$ It consists of a tiny CMOS image sensor, one solid tunable lens driven by MEMS thermal actuators and necessary housing structures, as illustrated in Fig. 6. The two MEMS thermal actuators are optimized by the finite-element method (FEM) and fabricated by the SOI MUMPs, while the lens elements are optimized by the ray-tracing technology and fabricated with the aforementioned diamond turning and replication process. The results show that the thermal actuator provides a maximum displacement of about $130 \mu \mathrm{m}$ with an input voltage of $10 \mathrm{~V}$, which tunes the focal length of the designed solid tunable lens from about 9.2 to about 7.9 $\mathrm{mm}$. Targets placed at various object distances are focused on by the image sensor individually by applying different voltages to the thermal actuators; this verifies the variable-focus capability of the camera module. Furthermore, the image quality of the lens within the whole tuning range is

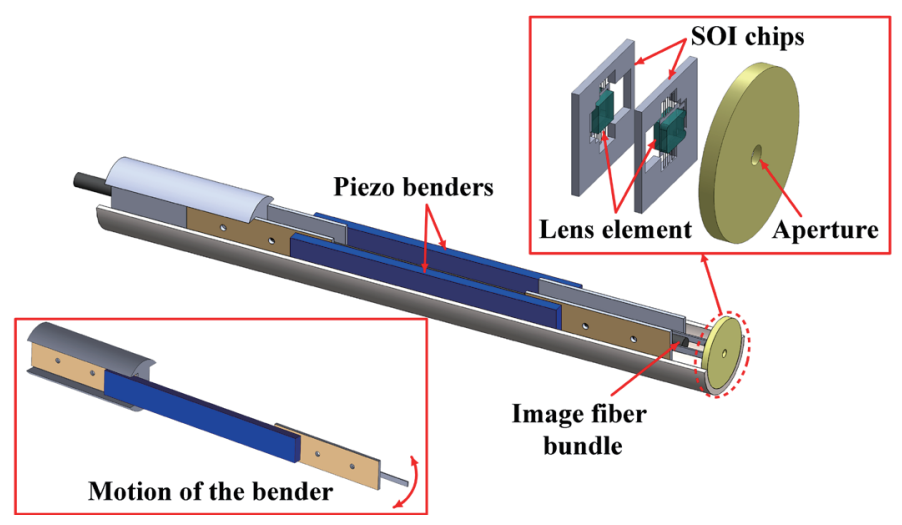

Fig. 5. (Color online) Schematic of the adjustablefocus endoscope based on the solid tunable lens.

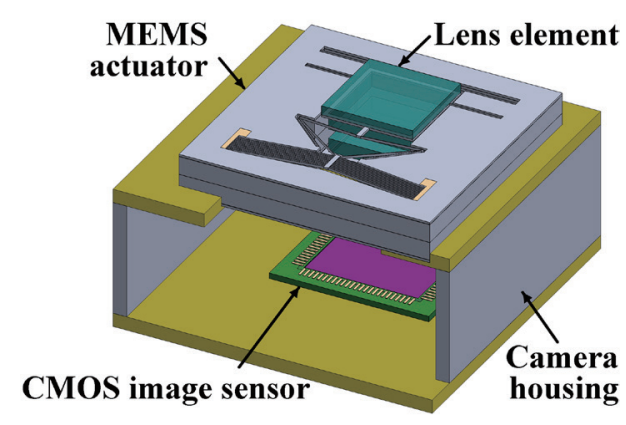

Fig. 6. (Color online) Schematic of the adjustablefocus camera module based on the solid tunable lens. 
found to be satisfactory. Such miniature variable-focus camera modules would be useful in various applications, including mobile phones, tablets, and surveillance systems.

Both examples demonstrate the potential of such solid tunable lenses in miniature applications, but the optical system design is relatively simple and only the function of adjustable focus is achieved. The future work in this field may include the further exploration of their applications in other miniature optical systems and the improvement of the optical performance with more complicated optical system designs.

\section{Conclusions}

In this paper, we reviewed the progress of solid tunable lenses based on the Alvarez-Lohmann principle. The progress of the modelling and design methods of such solid tunable lenses was summarized first. The results showed that the extended polynomials are more effective than the original cubic equation in the optimization process for optimum lens performance. In addition, the coefficients giving the minimum air volume between the two lens elements provided a good starting point for the optimization process. After that, the development of various prototypes of solid tunable lenses was illustrated referring to the examples in current literature. The driving mechanisms, performances, and practicability of all the prototypes were discussed. Lastly, the applications of such solid tunable lenses in miniature imaging systems were reviewed, showing their potential in different miniature applications.

Compared with liquid tunable lenses, the solid lenses show great advances, including stable performance against external environmental disturbance, ease of handling and assembly, and improved optical performance. It is considered that such solid tunable lenses have a promising future in various miniature applications.

\section{References}

1 C. P. Chiu, T. J. Chiang, J. K. Chen, F. C. Chang, F. H. Ko, C. W. Chu, S. W. Kuo, and S. K. Fan: J. Adhes. Sci. Technol. 26 (2012) 1773.

2 N. T. Nguyen: Biomicrofluidics 4 (2010) 031501.

3 H. M. Leung, G. Zhou, H. Yu, F. S. Chau, and A. S. Kumar: J. Micromech. Microeng. 20 (2010) 025021.

4 G. Zhou, H. M. Leung, H. Yu, A. S. Kumar, and F. S. Chau: Opt. Lett. 34 (2009) 2793.

5 S. H. Ahn and Y. K. Kim: Sens. Actuators, A 78 (1999) 48.

6 D. Y. Zhang, V. Lien, Y. Berdichevsky, J. Choi, and Y. H. Lo: Appl. Phys. Lett. 82 (2003) 3171.

7 A. Werber and H. Zappe: Appl. Optics 44 (2005) 3238.

8 N. Chronis, G. L. Liu, K. H. Jeong, and L. P. Lee: Opt. Express 11 (2003) 2370.

9 K. H. Jeong, G. L. Liu, N. Chronis, and L. P. Lee: Opt. Express 12 (2004) 2494.

10 D. Y. Zhang, N. Justis, and Y. H. Lo: Appl. Phys. Lett. 84 (2004) 4194.

11 M. Agarwall, R. A. Gunasekaran, P. Coane, and K. Varahramyan: J. Micromech. Microeng. 14 (2004) 1665.

12 L. Pang, U. Levy, K. Campbell, A. Groisman, and Y. Fainman: Opt. Express 13 (2005) 9003.

13 J. Chen, W. S. Wang, J. Fang, and K. Varahramyan: J. Micromech. Microeng. 14 (2004) 675.

14 G.-H. Feng and Y.-C. Chou: Sens. Actuators, A 156 (2009) 342.

15 C. C. Cheng, C. A. Chang, and J. A. Yeh: Opt. Express 14 (2006) 4101.

16 H. Yu, G. Zhou, F. S. Chau, F. Lee, and S. Wang: J. Micromech. Microeng. 18 (2008) 105017.

17 H. Oku, K. Hashimoto, and M. Ishikawa: Opt. Express 12 (2004) 2138.

18 S. T. Choi, J. Y. Lee, J. O. Kwon, S. Lee, and W. Kim: Proc. SPIE7208, MOEMS and Miniaturized Systems VIII, eds. D. L. Dickensheets, H. Schenk, and W. Piyawattanametha (SPIE Press, California, 2009) p. 72080P.

19 J.-Y. Lee, S.-T. Choi, S.-W. Lee, and W. Kim: Proc. SPIE7426, Optical Manufacturing and Testing VIII, eds. J. H. Burge, O. W. Fähnle, and R. Williamson (SPIE Press, California, 2009) p. 742603. 
20 S. W. Lee and S. S. Lee: Appl. Phys. Lett. 90 (2007) 121129.

21 C. A. Lopez, C. C. Lee, and A. H. Hirsa: Appl. Phys. Lett. 87 (2005) 134102.

22 H. Ren, D. Fox, P. A. Anderson, B. Wu, and S.-T. Wu: Opt. Express 14 (2006) 8031.

23 H. Ren and S.-T. Wu: Opt. Express 15 (2007) 5931.

24 B. Berge and J. Peseux: Eur. Phys. J. E 3 (2000) 159.

25 B. Berge: Proc. 18th IEEE Int. Conf. Micro Electro Mechanical Systems, 2005. MEMS 2005 (IEEE, New York, 2005) p.227.

26 B. Berge and J. Peseux: Eur. Phys. J. E 3 (2000) 159.

27 S.-K. Fan, C.-P. Chiu, and J.-W. Lin: Appl. Phys. Lett. 94 (2009) 164109.

28 C.-C. Cheng and J. A. Yeh: Opt. Express 15 (2007) 7140.

29 H. Ren and S.-T. Wu: Opt. Express 16 (2008) 2646.

30 H.-C. Lin, M.-S. Chen, and Y.-H. Lin: Trans. Electr. Electron. Mater. 12 (2011) 234.

31 H. T. Dai, Y. J. Liu, X. W. Sun, and D. Luo: Opt. Express 17 (2009) 4317.

32 Y. Choi, J.-H. Park, J.-H. Kim, and S.-D. Lee: Opt. Mater. 21 (2003) 643.

33 H. Ren, Y.-H. Fan, and S.-T. Wu: Opt. Lett. 29 (2004) 1608.

34 H. Ren and S.-T. Wu: Appl. Opt. 44 (2005) 7730.

35 H.-S. Ji, J.-H. Kim, and S. Kumar: Opt. Lett. 28 (2003) 1147.

36 J.-H. Kim and K. Satyendra: Jpn. J. Appl. Phys. 43 (2004) 7050.

37 D. Erickson, X. Heng, Z. Li, T. Rockwood, T. Emery, Z. Zhang, A. Scherer, C. Yang, and D. Psaltis: SPIE5908, Optical Information Systems III, eds. B. Javidi and D. Psaltis (SPIE Press, California, 2005) p. $59080 \mathrm{~S}$.

38 S.-Y. Lee, H.-W. Tung, W.-C. Chen, and W. Fang: IEEE Photonics Technol. Lett. 18 (2006) 2191.

39 G. Beadie, M. L. Sandrock, M. J. Wiggins, R. S. Lepkowicz, J. S. Shirk, M. Ponting, Y. Yang, T. Kazmierczak, A. Hiltner, and E. Baer: Opt. Express 16 (2008) 11847.

40 A. Santiago-Alvarado, S. Vazquez-Montiel, J. Munoz-López, V. M. Cruz-Martínez, G. Díaz-González, and M. Campos-García: J. Phys. Conf. Ser. 274 (2011) 012101.

41 P. Liebetraut, S. Petsch, J. Liebeskind, and H. Zappe: Light Sci. Appl. 2 (2013) e98.

42 L. W. Alvarez: USA Patent Two-Element Variable-Power Spherical Lens, No. 3,305,294 (1967).

43 A. W. Lohmann: Appl. Opt. 9 (1970) 1669.

44 L. W. Alvarez: USA Patent Variable-Power Lens and System, No. 3,507,565 (1970).

45 S. Barbero: Opt. Express 17 (2009) 9376.

46 Y. Zou, G. Zhou, Y. Du, and F. S. Chau: J. Opt. 15 (2013) 125711.

47 S. Barbero and J. Rubinstein: J. Opt. 13 (2011) 125705.

48 A. Grewe, M. Hillenbrand, and S. Sinzinger: Appl. Opt. 53 (2014) 7498.

49 Y. Zou, W. Zhang, F. S. Chau, and G. Zhou: Opt. Express 23 (2015) 20582.

50 S. Petsch, A. Grewe, L. Köbele, S. Sinzinger, and H. Zappe: Appl. Opt. 55 (2016) 2718.

51 A. Bauer, S. Vo, K. Parkins, F. Rodriguez, O. Cakmakci, and J. P. Rolland: Opt. Express 20 (2012) 14906.

52 K. Fuerschbach, J. P. Rolland, and K. P. Thompson: Opt. Express 19 (2011) 21919.

53 I. Kaya and J. P. Rolland: Adv. Opt. Technol. 2 (2013) 81.

54 I. M. Barton, S. N. Dixit, L. J. Summers, K. Avicola, and J. Wilhelmsen: Opt. Lett. 25 (2000) 1.

55 C. Huang, L. Li, and A. Y. Yi: Microsyst. Technol. 15 (2008) 559.

56 P. J. Smilie, B. S. Dutterer, J. L. Lineberger, M. A. Davies, and T. J. Suleski: Opt. Eng. 51 (2012) 013006.

57 J. Schwiegerling and C. Paleta-Toxqui: Appl. Opt. 48 (2009) 1932.

58 S. Rege, T. Tkaczyk, and M. Descour: Opt. Express 12 (2004) 2574.

59 A. N. Simonov, G. Vdovin, and M. C. Rombach: Opt. Express 14 (2006) 7757.

60 G. Zhou, H. Yu, and F. S. Chau: Opt. Express 21 (2013) 1226.

61 Y. Zou, W. Zhang, F. Tian, F. S. Chau, and G. Y. Zhou: Int. J. Nanotechnol. 12 (2015) 818.

62 Y. Zou, W. Zhang, F. Tian, F. S. Chau, and G. Y. Zhou: IEEE J. Sel. Top. Quantum Electron. 21 (2015) 100.

63 S. Schuhladen, F. Preller, R. Rix, S. Petsch, R. Zentel, and H. Zappe: Adv. Mater. 26 (2014) 7247.

64 Y. Zou, W. Zhang, F. S. Chau, and G. Zhou: Opt. Lett. 41 (2015) 1.

65 W. Zhang, Y. Zou, T. Lin, F. S. Chau, and G. Zhou: Proc. 8th Asia-Pacific Conf. Transducers and Micro/Nano Technology, Kanazawa, Japan (2016). 